\title{
REVIEW OF 510K APPROVAL PROCESS ON CLASS III DEVICES AND RELATED ISSUES
}

\author{
Chintalapati Jaya Sai Lakshmi Sowmya*,
} Harikaindukurib and Ravi Kumar Reddyjuturi

Department of Pharmaceutical Regulatory Affairs, Shri Vishnu College of Pharmacy (Autonomous), Vishnupur, Bhimavaram-534 202, Andhra Pradesh, India.

\begin{abstract}
In the USA, medical devices are regulated by the Food and Drug Administration (FDA) with an aim to ensure the safety and effectiveness of the devices. The Center for Devices and Radiological Health (CDRH) is an FDA section and looks after this procedure. Medical devices are classified into three categories based on the associated risk, namely: Class I, II and III. With respect to that, most Class I devices are exempted from $510(\mathrm{k})$ premarket notification submission, while most of Class II devices are submitted for premarket notification. On the other hand, Class III devices need to go through the Premarket Approval Application (PMA) and other class III devices, which are exempted from PMA must submit a 510(k) notification to the FDA. In the $510(\mathrm{k})$ procedure must demonstrate that the device is substantially equivalent to one legally marketed in the United States: (1) before May 28, 1976; or (2) to a device that has been determined by FDA to be substantially equivalent. To mark a device as substantially equivalent, manufacturers need to compare their device with one or multiple existing devices and provide suitable facts to support the claim. The base device selected for the comparison is called "predicate". It seems like the device and predicate are identical, but it's not. The $510(\mathrm{k})$ approved class III medical devices causing major harm/risk to the patients. This article reviews the pros and cons along with cases on the most commonly utilized FDA $510(\mathrm{k})$ regulatory pathway.
\end{abstract}

Keywords: Premarket approval, Medical device, predicates, recalls and review.

\section{INTRODUCTION}

The regulation of medical devices throughout the planet evolved at different times as a result of societal pressures. Within the United States, regulations began in the 1970 s after the government noticed 10,000 injuries frommedicaldevices.Duringthe same era, the Dalkon Shield intrauterine devices caused 200 septic second-trimester abortions and 11 maternal deaths. These events led to the Medical Device Amendments of 1976, which gave the US FDA the authority to regulate medical devices. ${ }^{1}$

FDA is liable for assuring the "safety and effectiveness" of all medical devices, the Food and Drug Administration (FDA) regulates device manufacturers' ability to market devices within the US. How the FDA carries out this task, is debatable. Consumer protection advocacy groups demand tighter regulatory oversight, at the same time many physicians and some patient advocacy groups argue that the current regulations decrease innovation and prevent the patient to use novel devices. ${ }^{2,3}$ Within the FDA, the Centre for Devices and Radiological Health (CDRH) has primary responsibility for the premarket assessment of the latest medical technology. The CDRH not only considers the risks of new devices but also monitors them via a nationwide 
post-market surveillance system. New devices are classified as low (I), moderate (II), or high risk (III). Among the thousands of device applications submitted annually, fewer than a hundred those ponder to be novel and high-risk endure a premarket approval application (PMA). Most of the new device applications are also initiated via a premarket notification application [510(k)], the FDA process based on the assumption that most devices are essentially equivalent to those already approved. For the 510(k) pathway, the manufacturer may not be required to provide safety and efficacy data. Annually more than $90 \%$ of medical devices are approved through the $510(\mathrm{k})$ process whereas less than $10 \%$ are approved through the PMA process. At the same time, higher recall rates for devices are cleared through the $510(\mathrm{k})$ process compared with those approved via the PMA process.

As a consequence of the above scenario in the approval process of medical devices through 510k, some serious events are recorded in the past 10 years. In this regards
US FDA issued several guidelines, but still there isa number of issues with $510(\mathrm{k})$ which is discussed in this paper.

\section{METHODS}

\section{Medical device classification}

Devices are classified into 3 groups by the FDA: Class I or "low risk of illness or injury" (e.g., surgical gauze); Class II or "moderate risk" (e.g., suture); and sophisticated Class III, those which "support to sustain the human life (e.g., pacemakers). Class I and II devices are subject to less stringent regulatory approval processes than Class III devices. ${ }^{4}$

\section{Basic Pathways to Medical Device Approval in the US}

Step 1: Classify the device

Step 2: Select the suitable pre-market submission pathway

Step 3: If the device requires a full PMA

Step 4: Prepare the appropriate application

Step 5: Send the submission to the FDA

Step 6: Record the establishment and list the device $^{5}$

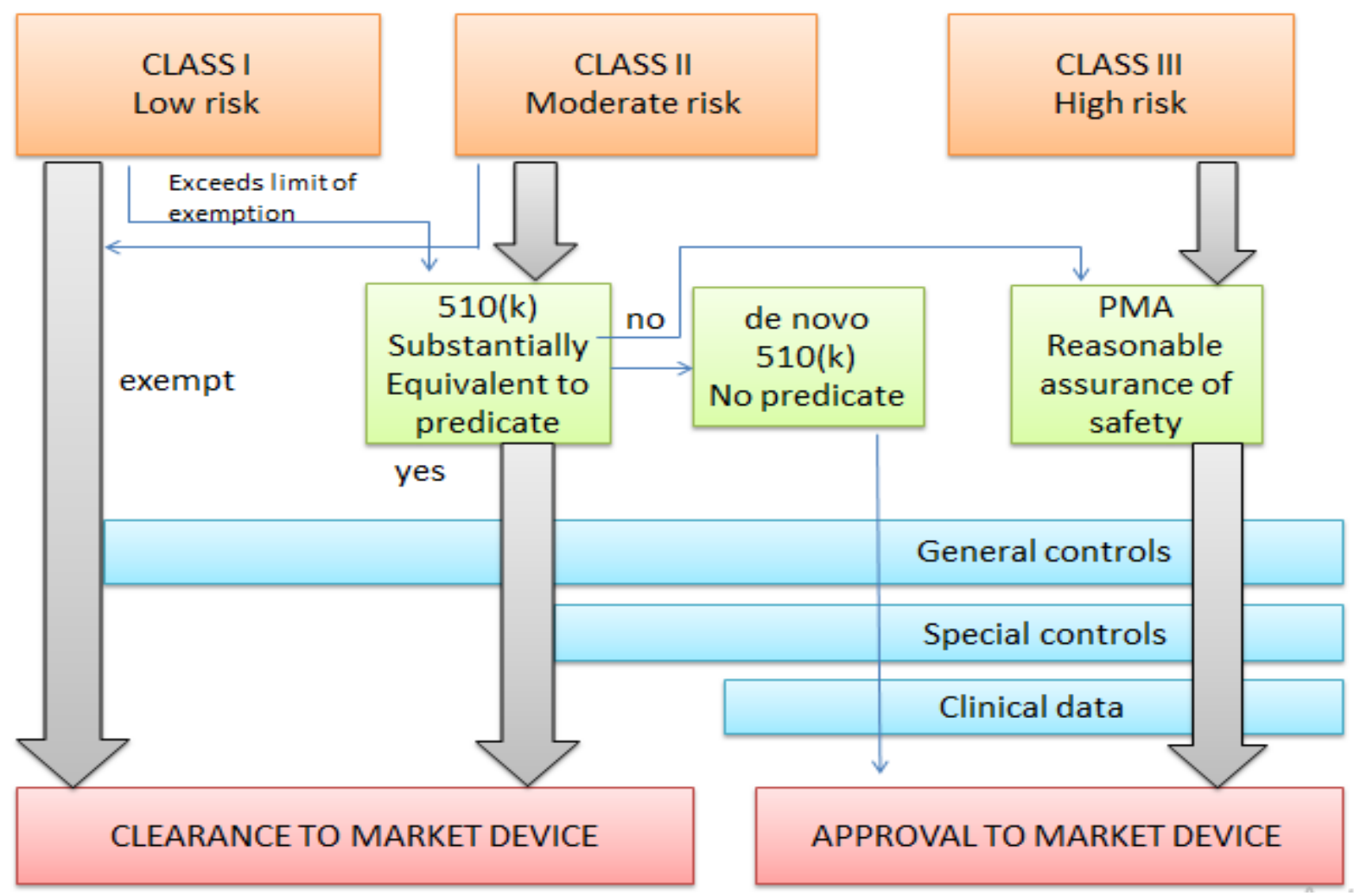

Fig. 1: Flow chart of medical device approval procedure

Fig. 1: flow chart of medical device approval procedure in the US. Drawn in PowerPoint windows 2010 
marketed is no less than as safe and effective, that is, well equivalent, to a legally marketed device (21 CFR 807.92(a)(3)) which is not subjected to PMA. Submitters must compare their device to one or more similarly and legally marketed devices to make as well as support their substantial equivalency claims. A lawfully marketed device is a device that was marketed before 28 May 1976 (pre amendments device) or a device which has been reclassified from Class III to class II or I, a tool that has been found SE through the $510(\mathrm{k})$ process, or a device that was granted marketing authorization via the De Novo classification process under section 513(f) (2) of the FD\&C Act that is not exempt from premarket notification requirements. The lawfully marketed device(s) to which equivalence is drawn is often referred to as the "predicate." Although devices recently cleared under $510(\mathrm{k})$ are often selected as the predicate to which equivalence is claimed, any lawfully marketed device could also be used as a predicate. Legally marketed also means that the predicate cannot be one that is in violation of the Act. $^{[6]}$

Until the submitter receives an order declaring a device SE, the submitter could not proceed to market the device. Once the device is set to be SE, it could then be marketed within the U.S. The SE determination is typically created in no more than 90 days and is formed upon the data submitted by the submitter.

\section{DISCUSSION}

\section{Review on 510k Approval Process\& its} Implications

The following are the deviations from the approval process by the manufacturers in the US:

The $510(k)$ is so far the most commonly utilized FDA regulatory pathway. Furthermore, devices cleared by $510(\mathrm{k})$ most often do not require supporting empirical clinical research.
It requires bench trial studies. While this simplifies, and cheapens, the process for a manufacturer, it diminishes "real-world" testing prior to widespread dissemination and implantation of the product. ${ }^{7}$

Generally, Class III devices were meant to undergo the more rigorous premarket approval (PMA), the only pathway that requires clinical data. However, class III devices were allowed to receive a review for substantial equivalence temporarily, until the FDA classified these devices or broadcast regulations requiring PMA. Congress had always anticipated class III devices to undergo PMA, and in 1990, it directed the agency to determine a schedule to finish the transition to PMAs for all devices that were to remain in class III. ${ }^{[8]} \mathrm{As}$ of December 19, 2012, the FDA still had not completed this transition to PMA for high-risk devices, although it had stated its intention to clear proposed rules for all left over class III pre-amendment devices by December 31 , $2012 .{ }^{[9]}$ Currently, 19 different types of class III devices are allowed to reach patients through $510(\mathrm{k})$ clearance. Because of this loophole, companies that market these devices are often legally able to obtain clearance without demonstrating safety and effectiveness through clinical studies, but by claiming substantial equivalence to earlier "predicate devices" or pieces of those devices which may also have been found substantially equivalent to even earlier devices, and so on. ${ }^{10}$

As many predicates have been recalled from the market for some safety reasons, an FDA finding of substantial equivalence does not mean that a new device is safe and effective; it means only that the device is deemed no less safe and no less effective than a predicate. Even voluntarily recalled devices can serve as predicates, as long as the FDA did not formally remove these devices from the market or a court did not find them adulterated or misbranded. 


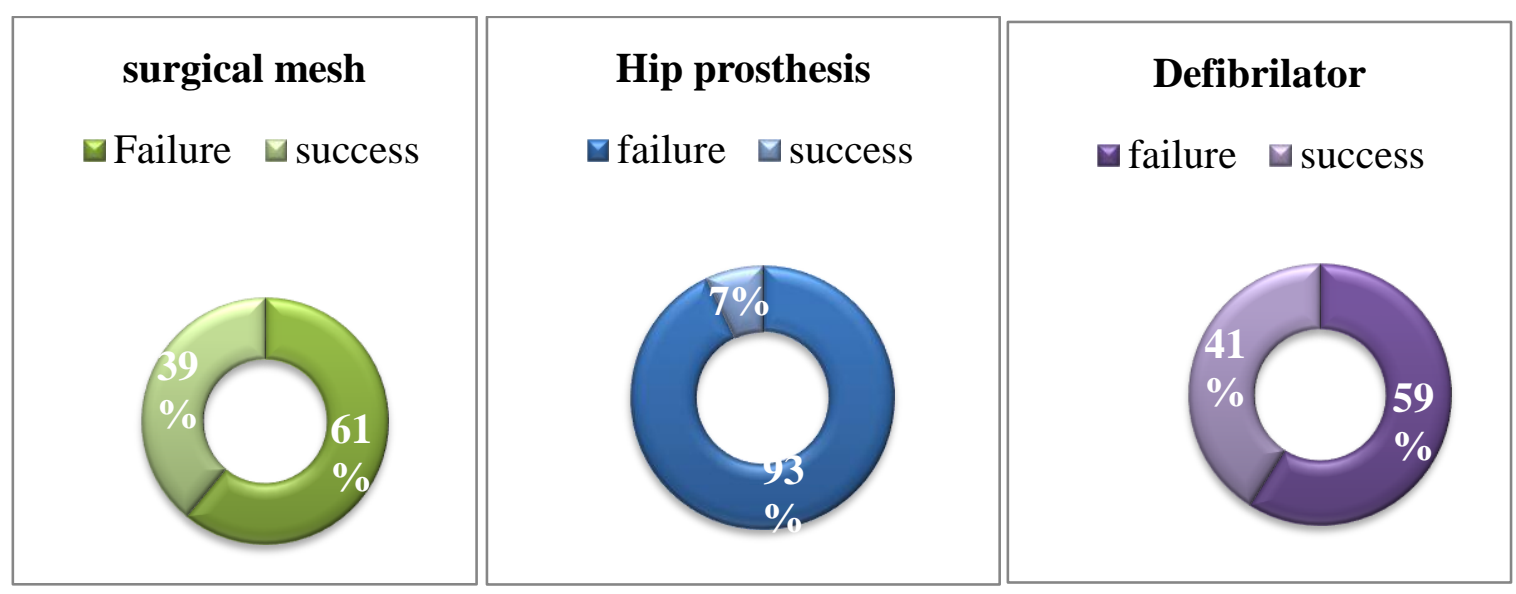

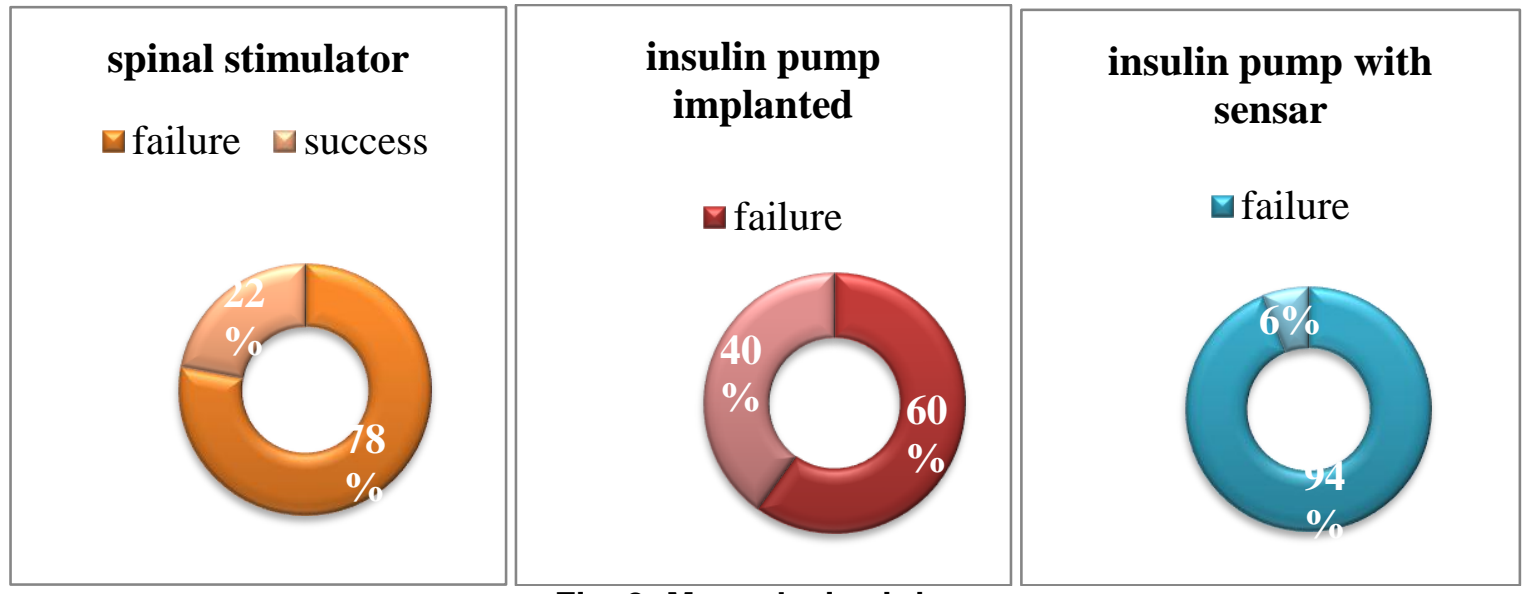

Fig. 2: Many device injury report stem from a few devices

Fig. 2: Although there are roughly 4,000 types of medical devices in the FDA data, six of them accounted for which are implanted in 50,000-60,000 people a year among them more than $50 \%$ of devices are reported for injuries. ${ }^{11}$ 
510(k) clearance on metal-on-metal hip implants, such as the DePuy ASR XL Acetabular Cup System, which received FDA clearance in July 2008 without a clinical study. The $510(\mathrm{k})$ clearance for the ASR XL mainly concentrated on 3 characteristics:

1. The porous bone ingrowth surface

2. Metal-on-metal articulation

3. Large femoral head sizes (57 to $63 \mathrm{~mm}$ ), which were larger than those of the predicate total hip prostheses

These three characteristics were uniquely combined in the ASR XL but were evaluated for "substantial equivalence" by comparing selected characteristics to different predicate devices, none of these devices contained all the above characteristics (i.e., they were "split predicates"). ${ }^{10}$

In most cases, the predicates were not metal-on-metal and were substantially different in design from the ASR XL. None of the predicates in the ancestry had the same combination of features as the ASR XL acetabular component. Approval of the large metal-on-metal articulation was based on a much minor group of predicates, some of which differed substantially in design from the ASR XL or had a poor clinical performance. Ultimately, clearance was based on the claim that these predicate devices were substantially equivalent to three prostheses that were used before 1976: the McKee-Farrar, Ring, and Sivash metal-on-metal total hip prostheses. It is necessary to notice that these 3 devices were discontinued way back (and well before clearance of the ASR XL) as a result of their risk of revision was higher than that of other hip prostheses. ${ }^{10,12,13}$

However, this hip varied substantially in design from the ASR XL in two major ways. The cup wasn't solid metal but instead consisted of a metal shell and a metal articular surface inlay with a polyethylene "sandwich" between the two. A second distinction was head size: the Metasul had a lot of smaller heads $(\leq 32 \mathrm{~mm})$ than the ASR XL. The use of larger heads was a vital characteristic of the ASR XL. The clearance for the large metal heads with sleeves was based in part on predicates that were not used in total hip replacement but were designed for use in partial hip replacement, in which the large metal heads articulate with the natural articular cartilage of the acetabulum, not with a metal cup. Due to the friction between the two metal surfaces fine particles were released from the implant and get attached to the soft tissues in the body. This ancestry reveals serious flaws in the $510(\mathrm{k})$ procedure for metal-on-metal hips, which resulted in the clearance of a new device that was never shown to be safe and effective. A clinical trial might have identified the highrevision rate of the ASR.As thousands of people undergone the painful procedure and suffered a lot by unknown risks with this device that entered the market without clinical trials $^{10}$

\section{Transvaginal Mesh}

Transvaginal mesh is a net-like implant accustomed to treat stress urinary incontinence (SUI) and pelvic organ prolapse (POP). Doctors deem transvaginal mesh to strengthen a weakened vaginal wall or support the urethra or bladder neck. Medical device manufacturers noticed the clinical practice and responded by creating a mesh product specifically designed to treat SUI and repair POP. The FDA cleared the first mesh for SUI in 1986.

In December 2017 a review published in the British Medical Journal openly revealed that 61 manufacturers sold mesh implants without a clinical trial. According to the review, manufacturers were able to get their devices on the market by using the FDA approval process called $510 \mathrm{k}$.

In the case of transvaginal mesh major parts of devices were cleared for the sale counting on 2 devices- Ethicon Mersilene mesh and Boston scientific protegenslinc (recalled in 1999 for some safety issues).

FDA determines that serious adverse events are reporting from 2008. In January 2008 the agency have received 69000 adverse events are reported. The report indicates that they are 64000 cited injuries and 393 deaths.

Since 2008 serious adverse events are reporting FDA did not take action on device approval process and FDA recalled many devices from the market for some safety reasons but the manufacturer sold the product by changing the name and use of the device and again went for the 510k approval process and placed in the market in the following years. Even though the device is banned in 1999 it is still being used as a predicate and the family tree is continuing still in $2019 .{ }^{14}$ 


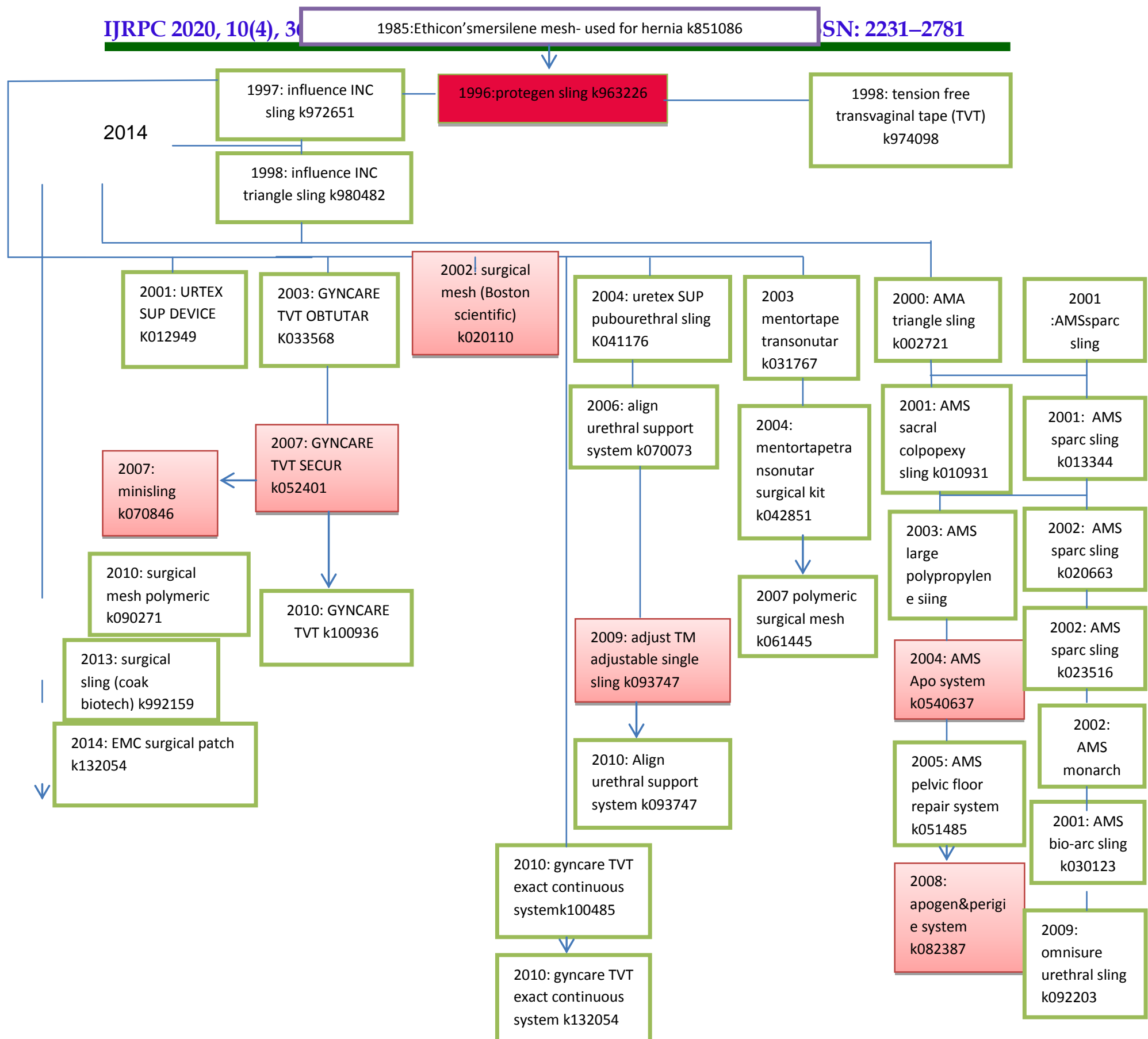

Fig. 3: FDA chain approval for transvaginal mesh through

$510 \mathrm{k}$ using protegen sling as a predicate device

Fig. 3: shows that the chain of device approvals using 'substantial equivalence Newer devices are compared with various older devices, and devices with similar names are compared with each other. Some devices have been removed from the market and some have had changes to their indications over time. ${ }^{15}$

Doctors performed 2, 60, 000 mesh surgeries for urinary incontinence, 3, 00,000 surgeries for POP. Most of the people face Complications which include, bleeding, 
infection, mesh erosion, nerve damage, neuromuscular problems, organ perforation, vaginal shrinkage,Vaginal mesh erosion require multiple surgeries to correct and may result in continuous pain after the mesh is removed. Thousands of women have filed lawsuits against mesh manufacturers, claiming the devices caused serious complications. So far, several device companies, including Johnson \& Johnson and Boston Scientific have agreed to pay about $\$ 8$ billion to resolve more than 100,000 claims, The New York Times reported in the year 2019 .

\section{Knee implant}

More than 600,000 people in the U.S. undergo a partial or total knee operation every year. Knee implant manufacturers are responsible for the appropriate marketing of their products. They must warn consumers and health care professionals about their devices' risks. Manufacturers often rely on the agency's Premarket Notification process. This is also called the $510(k)$ process, it lets knee replacement manufacturers skip additional rigorous tests. They only have to show their device is "substantially equivalent" to similar products.

Annually more than $50 \%$ of approved knee implants are recalled by the FDA because these devices involve defective parts that pose a risk of serious injuries, such as implant rejection, loosening, instability, dislocation, misalignment may be due to mechanical problems or defects with the implant. More serious complications need to undergo revision surgery to correct. There is also the risk of death from total knee replacement surgery. The American Association of Hip and Knee Surgeons say that 90 to 95 percent of knee replacements will last 10 years. ${ }^{16}$

\section{DePuy Attune Knee System History}

In Dec 2010, the DePuy attune Knee System was approved by the U.S. Food and Drug Administration under the agency's 510(k) approval process.

This is a special expedited approval process in which a manufacturer must prove that the device is "substantially equivalent" to a product already in the market legally. According to the FDA, "a claim of substantial equivalence does not mean the new and predicate devices must be identical." A device must only be equivalent in design, use, and others.

Devices approved by $510(\mathrm{k})$ do not need to conduct clinical trials or offer proof of safety before approval.

In the application, DePuyOrthopedics said the DePuy attune Knee System as well akin to eight different products in the market at the time, like the sigma Patella and Zimmer NexGen CR Knee System (which is not in use).

Not long after the DePuy attune Knee System was approved, the FDA began receiving reports of failure within the devices. "In most cases, the patients who experience a premature failure of their attune Knee System is to endure a revision surgery to get rid of the implant and replace it. "These revision surgeries are usually very painful and invasive than the initial replacement surgery."

Thousands of patients have already been implanted with the Attune device. Some had to undergo complicated revision surgery to correct the premature failure of the device. Not only attune device but many knee implants, manufactured by different companies were creating such serious adverse events. Still, the FDA didn't take any action on the approval process.

\section{Why manufacturers mostly rely on 510k approval process rather than PMA}

Premarket approval (PMA) is the FDA process of scientific and regulatory review to judge the safety and effectiveness of class III medical devices. Class III devices are those which support or sustain human life, are of significant importance in preventing impairment of human health, or which present a possible, unreasonable risk of illness or injury. Due to the level of threat associated with Class III devices, FDA has determined that general and special controls alone are insufficient to assure the safety and effectiveness of the class III devices. Therefore, these devices require a premarket approval (PMA) application under section 515 of the FD\&C Act so as to get marketing approval. A PMA is the strictest device marketing application and is required by the FDA for any new device In the PMA; a device must be shown to have sufficient scientific evidence that it is safe and effective in its intended use by conducting the clinical trials. ${ }^{18}$

It costs a manufacturer an average of about $\$ 94$ million to bring a medical device onto the market through PMA, compared to $\$ 31$ million for the much less stringent $510(\mathrm{k})$ process. Part of the cost is in the form of a user fee from the FDA, with the standard fee for PMA set at nearly $\$ 235,000$, compared to $\$ 4,690$ for $510(k){ }^{19}$

PMA requires extensive data from laboratory and clinical studies, which aren't required under the more lenient $510(\mathrm{k})$ process. In the US Annually more than $90 \%$ of medical devices are approved through the $510(\mathrm{k})$ 
process whereas less than $10 \%$ are approved through the PMA process. ${ }^{20}$

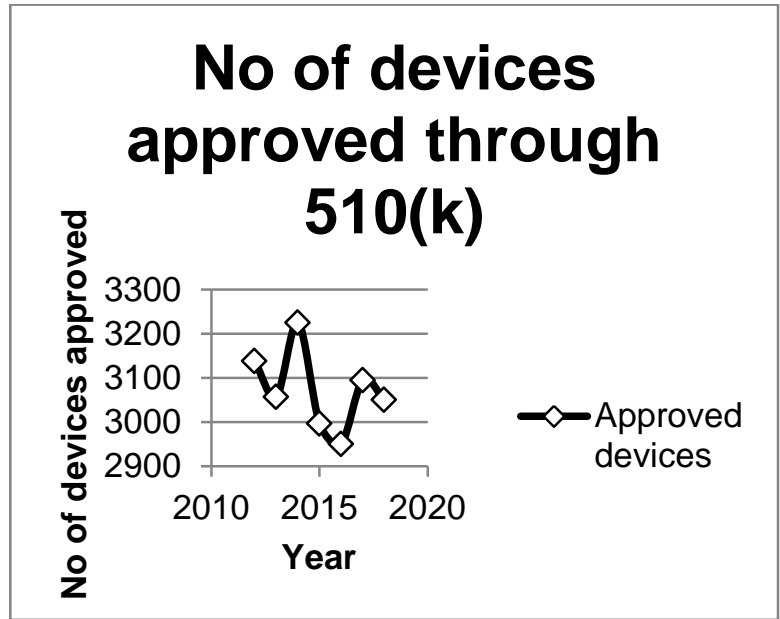

Fig. 4: no of devices approved through $510(k)$ annually. ${ }^{20}$

Percentage of medical devices approved annually

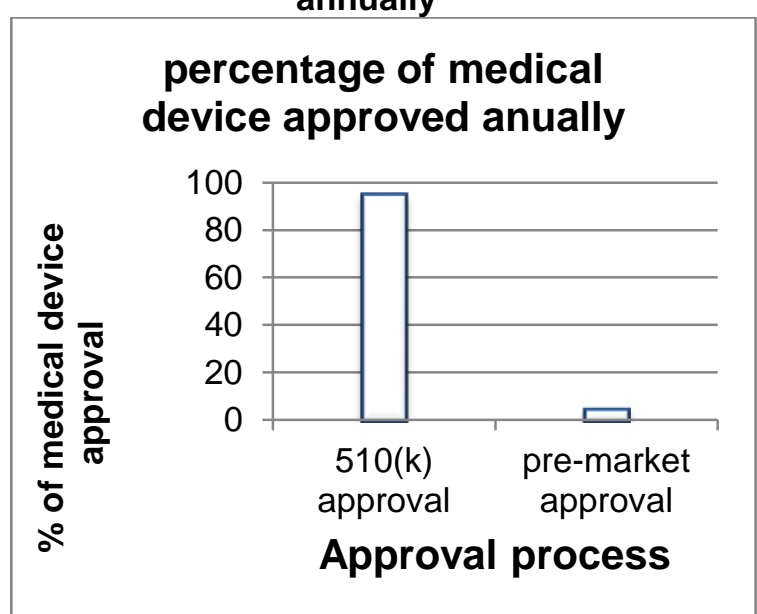

Fig. 5: Percentage of devices approved through 510(k) annually

Recalls on $\mathbf{5 1 0}(k)$ approved class III devices

Classification

Recalls are classified into a numerical designation (I, II, or III) by the FDA to indicate the relative degree of health hazard presented by the product being recalled.

$\checkmark$ Class I - a situation in which there is a reasonable probability that the use of, or exposure to, a product will cause serious adverse health consequences or death.

$\checkmark$ Class II - a situation in which use of, or exposure to, a product may cause temporary or medically reversible adverse health consequences or where the probability of serious adverse health consequences is remote.

$\checkmark$ Class III - a situation in which use of, or exposure to, a product is not likely to cause adverse health consequences. ${ }^{21}$

\section{Recalled devices}

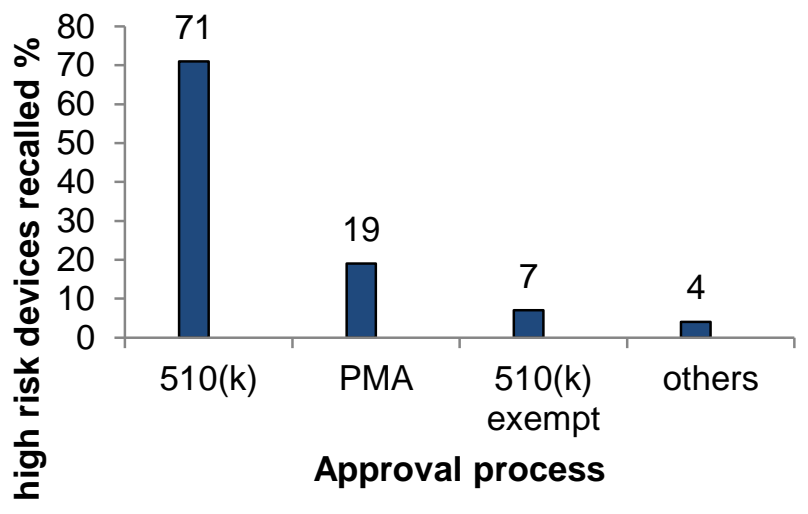

Fig. 6: High-risk recalled devices classified by how the US Food and Drug Administration reviewed them from 2005 through 2019. PMA indicates premarket approval; 510(k), the less stringent premarket notification process.

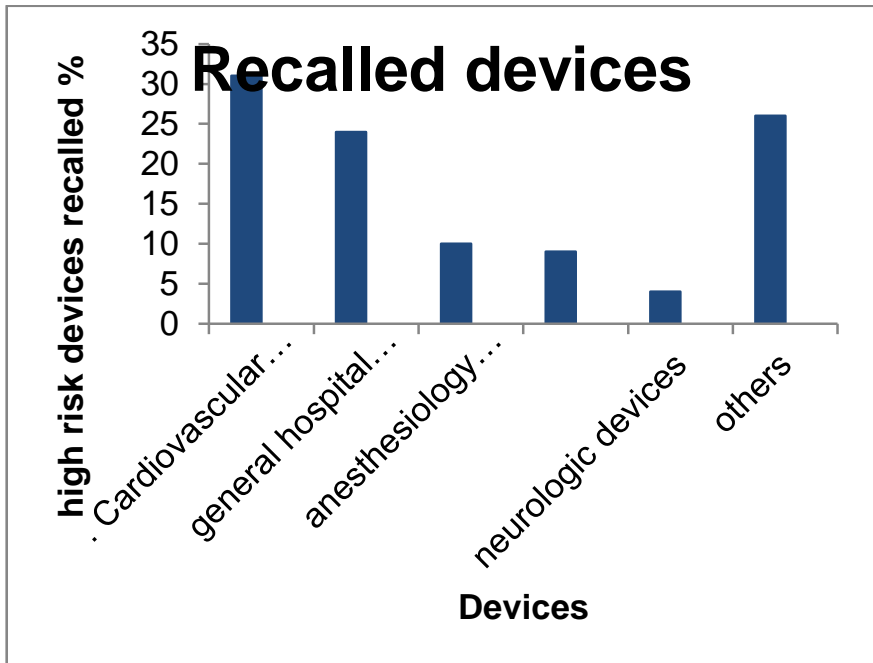

Fig. 7: High-risk recalled devices categorized by medical specialty designated by the US Food and Drug Administration. ${ }^{22}$

Table 1: High-Risk Recalls of Class III Devices Cleared Through 510(k)

\begin{tabular}{|c|c|c|c|}
\hline Year & Device name & $\begin{array}{c}\text { Type of } \\
\text { recall }\end{array}$ & $\begin{array}{c}\text { Approval } \\
\text { procedure }\end{array}$ \\
\hline 2019 & Transvaginal mesh & Class I & $510 \mathrm{~K}$ \\
\hline 2017 & Eliquirs & Class I & $510 \mathrm{~K}$ \\
\hline
\end{tabular}




\begin{tabular}{|c|c|c|c|}
\hline 2014 & $\begin{array}{c}\text { Ethicon Gynecare } \\
\text { Morecellex }\end{array}$ & Class I & $510 \mathrm{~K}$ \\
\hline 2010 & $\begin{array}{c}\text { Depuy ASR Hip } \\
\text { Implant }\end{array}$ & Class I & $510 \mathrm{~K}$ \\
\hline 2009 & Yaz & Class I & $510 \mathrm{~K}$ \\
\hline 2008 & $\begin{array}{c}\text { AED 10 and MRL } \\
\text { Jumpstart AED }\end{array}$ & Class I & $510 \mathrm{~K}$ \\
\hline 2007 & AED 10 & Class I & $510 \mathrm{~K}$ \\
\hline 2006 & AED 20 & Class I & $510 \mathrm{~K}$ \\
\hline 2005 & Kugel Hernia Patch & Class I & $510 \mathrm{~K}$ \\
\hline
\end{tabular}

The FDA includes 115 names of recalled devices on their high-risk recall list. Of these 115 recalls, the FDA designated 113 as class I recalls, for example, the FDA received reports of 116086 potential device-related injuries, 2830 potential device-related deaths, and more than 200000 adverse events reports concerning medical devices. ${ }^{23}$ The PMA process was used to approve 21 of 115 devices listed as high-risk recalls that could cause serious health problems or death $(19 \%) .{ }^{24}$ Eight were cleared through the 510(k) process. Cardiovascular devices covered the largest recall category, with 35 separate recalls accounting for $31 \%$ of devices on the FDA's high-risk recall list. Two-third of these recalled cardiovascular devices were cleared by the $510(\mathrm{k})$ process $(66 \%)$, while $34 \%$ were approved through the PMA process. All of those recalled devices were used for treating cardiovascular disorders. Most were automated external defibrillators (AEDs) approved for the resuscitation of patients in cardiac arrest. The researcher has reported that more than $20 \%$ of just above 1 million AEDs in circulation were recalled by the FDA, and hundreds of people died because of AED malfunctions. ${ }^{25}$

The second-largest high-risk recall group (24\% of the total) was made up of general hospital devices, including insulin pumps, intravenous infusion devices, and patient's lifts. Seventyfour of these recalled devices were cleared through $510(\mathrm{k})$ review randomly $22 \%$ were approved through the rigorous 11PMA process. The third-largest high-risk recalled category ( $10 \%$ of the total) was anesthesiology devices, including mechanical ventilators. All of those devices were cleared by the $510(k)$ process. The fourth and fifth largest groups of high-risk recalls were clinical chemical analysis and neurologic devices, respectively. Nine percent of fall recalls were clinical chemical analysis devices such as glucose meters and other diagnostic testing equipment. These devices were cleared by the $510(k)$ process. The present analysis demonstrates that most of the medical devices recalled by the FDA owing to serious risks during the past five years were approved through the $510 \mathrm{k}$ regulatory process or were completely exempted from regulatory review
$(78 \%)$. As such, these devices didn't undergo clinical testing or premarket inspections, nor were post-market studies required to see safety and efficacy. While even the more rigorous PMA criteria for device approval are often scientifically inadequate to ensure patient safety, PMA standards are clearly superior to $510(\mathrm{k})$ standards. Of the recalled devices cleared for the market through the $510(\mathrm{k})$ process, $12 \%$ were marketed for risky or life-sustaining class III indications, which are required by law to undergo a full PMA regulatory review.

\section{CONCLUSION AND RECOMMENDATIONS}

Thousands of devices reach the market annually in the US among them class III devices play a significant role which helps to sustain human life, nevertheless, these devices enter into the market without providing adequate clinical data. Two third of the Medical devices which are approved through the less rigorous $510 \mathrm{k}$ process is recalled due to major harm/death caused to the patients. Examples of such devices are Metal-hip-metal implant, transvaginal mesh, knee implant.

Number of high-risk recall for medical devices and the number of patients affected by these devices would be decreased if certain changes are made

1. If the FDA fully implements current law that subjects lifesaving and lifesustaining (class III) devices to PMA process

2. The FDA expands the use of their authority to inspect the manufacturing of $510(\mathrm{k})$ devices most frequently, similar to that of the devices approved through the PMA process

3. The FDA should also consider special controls in the $510(\mathrm{k})$ approval process as they follow for the PMA process,

suchasperformancestandards, and product-specific characteristics.

4. FDA definition for class III devices should also include potential risk devices along with high-risk devices.

\section{ABBREVATIONS}

FDA- Food and Drug Adminstration.

\section{CONFLICTS OF INTEREST}

Authors declare no conflict of interest.

\section{REFERENCES}

1. Maak TG and Wylie JD. Medical device regulation: a comparison of the United States and the European Union. Journal of the American 
Academy of Orthopedic Surgeons. 2016; 24(8):537-43.

2. Lauer $M$, Barker JP, Solano $M$ and Dubin J. FDA Device Regulation. Missouri medicine. 2017;114(4):283.

3. Public Citizen. Substantially Unsafe: Medical Devices Pose Great Threat to Patients; Safeguards must be Strengthened, Not Weakened. https://www.citizen.org/documents/sub stantially-unsafe-medical device report. pdf2012.

4. US Food and Drug Administration: Medical devices: Classify your medical device: https://www.fda.gov/medicaldevices/overview-deviceregulation/classify-your-medicaldevice. [Accessed on October 20, 2019].

5. Van Norman GA. Drugs, devices, and the FDA: part 2: an overview of approval processes: FDA approval of medical devices. JACC. Basic to Translational Science. 2016;1(4):27787.

6. US Food and Drug Administration: Medical devices: Pre-Market Notification $510 \quad$ (k): https://www.fda.gov/medicaldevices/premarketsubmissions/premarket-notification510k. [Accessed on October 21, 2019].

7. Healthy news review. Gary schwitzer: Why 'approved' medical devices in the U.S. may not be safe or effective: https://www.healthnewsreview.org/tool kit/tips-for-understandingstudies/medical-devices/.[Accessed on October 22, 2019].

8. Challoner DR and Senate US. Medical devices and the public's health: the FDA $510(\mathrm{k})$ clearance process at 35 years. Written Statement before the Committee on Health, Education, Labor, and Pensions US Senate, Institute of Medicine of the National Academics, Washington. [Accessed on October 23, 2019].

9. US Food and Drug Administration: Medical devices: FDA forms: https://www.fda.gov/about-fda/reportsmanuals-forms/forms. [accessed on October 23, 2019].

10. Ardaugh BM, Graves SE and Redberg RF. The $510(\mathrm{k})$ ancestry of a metalon-metal hip implant. New England Journal of Medicine. 2013;368(2):97100.

11. Stat news. Mitch Weiss and Holbrook Mohr:Medical devices for pain, other conditions have caused more than 80,000 deaths since 2008 by associated press November 25, 2018: https://www.statnews.com/2018/11/25/ medical-devices-pain-otherconditions-more-than-80000-deathssince-2008/.[Accessed on october25, 2019].

12. Ring PA. Ring UPM total hip arthroplasty. Clinical orthopaedics and related research. 1983;(176):115-23.

13. SCabitza P and Percudani W. Longterm results of 2 continuous series of total hip prostheses of the McKeeFarrar metallo-metal type and the Charnleymetallo-plastic type. Archivio per le scienzemediche. 1979;136(1):45-50.

14. Drug watch. Michelle Llamas:Transvaginal Mesh: Https://www.drugwatch.com/transvagi nal-mesh/. [Accessed on October 26, 2019].

15. Heneghan CJ, Goldacre B, Onakpoya I, Aronson JK, Jefferson T, Pluddemann A and Mahtani KR. Trials of transvaginal mesh devices for pelvic organ prolapse: a systematic database review of the US FDA approval process. BMJ open. 2017;7(12):e017125.

16. Drug watch. Terry Turner:Knee Replacement Recalls: https://www.drugwatch.com/kneereplacement/recalls/. [accessed on October 27,2019].

17. Drug watch. TerryTurner:DePuy Attune Knee Replacements: https://www.drugwatch.com/kneereplacement/depuy-attune/. [Accessed on October 26].

18. US Food and Drug Administration, Medical devices: Premarket Approval (PMA): https://www.fda.gov/medicaldevices/premarketsubmissions/premarket-approval-pma. [Accessed on October 25, 2019].

19. Drug watch. Elaine Silvestrini: Premarket Approval (PMA): https://www.drugwatch.com/fda/prema rket-approval/ . [Accessed on October 27, 2019].

20. Emergo. How long it takes the US FDA to clear medical devices via the $510(\mathrm{k})$ process. https://www.emergogroup.com/sites/d efault/files/emergo-fda-510k-dataanalysis-2017.pdf. [Accessed on 28 October, 2019]. 
21. US Food and Drug Administration, Medical devices: Recalls, Corrections and Removals (Devices): https://www.fda.gov/medicaldevices/postmarket-requirementsdevices/recalls-corrections-andremovals-devices. [Accessed on October 30, 2019].

22. Zuckerman DM, Brown $P$ and Nissen SE. Medical device recalls and the FDA approval process. Archives of internal medicine. 2011;171(11):100611. [Accessed on October 21, 2019].

23. US Food and Drug Administration, Center for Devices and Radiological Health. CDRH FY 2006 highlights: www.fda.gov/downloads/AboutFDA/C entersOffices
CDRHReports/ucm129258.pdf. [Accessed October 31, 2019].

24. US Food and Drug Administration. Medical devices: list of device recalls: http:

//www.fda.gov/MedicalDevices/Safety/ RecallsCorrectionsRemovals/ListofRe calls /default.htm. [Accessed on November 1, 2019].

25. Maisel WH. Medical devices: are current regulations doing enough for patients? testimony before the Subcommittee on Health, Committee on Energy and Commerce, House of Representatives: June 2009. http: // energy commerce. house.gov/index.php?option=com_con tent\&view=article\& id. [Accessed on November 2, 2019]. 